\title{
Los efectos de la pandemia de COVID-19 en el ecosistema de festivales cinematográficos de la Comunitat Valenciana
}

\author{
Samuel Fernández-Pichel | snferpic@acu.upo.es \\ Centro Universitario Internacional - Universidad Pablo de Olavide \\ Irene Liberia Vayá | iliberia@us.es \\ Universidad de Sevilla \\ Sara Mansanet Royo| Sara.mansanet@fundacions.uv.es \\ Universitat de València
}

Palabras clave

"festival de cine"; "cine"; "COVID"; "pande-

mia"; "Comunitat Valenciana"

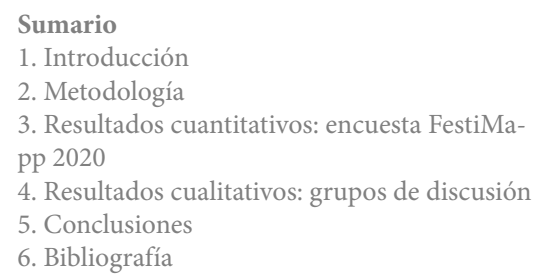

Sumario

1. Introducción

2. Metodología

3. Resultados cuantitativos: encuesta FestiMapp 2020

4. Resultados cualitativos: grupos de discusión

5. Conclusiones

6. Bibliografía

\section{Resumen}

Este artículo analiza cómo ha afectado la pandemia de COVID-19 a los festivales de cine de la Comunitat Valenciana en un contexto de evolución forzada de este sector cultural a escala global. Para ello, se ha llevado a cabo, en primer lugar, un trabajo cuantitativo basado en una encuesta telemática enviada a la totalidad de festivales censados en el territorio valenciano. En segundo término, me-

diante la técnica de investigación cualitativa del grupo de discusión, se ha profundizado en los datos y conclusiones extraídas de la primera parte del estudio. Los principales resultados indican que, con variaciones respecto a las fechas habituales, reducción de aforos y programaciones, y la adopción de las medidas higiénico-sanitarias pertinentes, la inmensa mayoría de los festivales pudieron celebrar su edición de 2020 y, pese a las notables diferencias entre los de gran formato y aquellos con una envergadura menor, todos ponen el foco en la vuelta a la presencialidad, aunque aplicando los aprendizajes del último año. Además del objetivo de regresar cuanto antes a la situación pre-COVID, la pandemia ha puesto de manifiesto la necesidad apremiante de colaboración entre certámenes, acelerando la creación de la Asociación de Festivales de Cine de la Comunitat Valenciana.

\section{Cómo citar este texto:}

Samuel Fernández-Pichel, Irene Liberia Vayá, Sara Mansanet Royo (2021): Los efectos de la pandemia de COVID-19 en el ecosistema de festivales cinematográficos de la Comunitat Valenciana, en Miguel Hernández Communication Journal, Vol. 12 (2) pp. 539 a 563 . Universidad Miguel Hernández, UMH (Elche-Alicante). DOI: 10.21134/mhjournal.v12i.1357 


\title{
The impact of the COVID-19 Pandemic on the Valencian Community's film festival ecosystem
}

\author{
Samuel Fernández-Pichel | snferpic@acu.upo.es \\ Centro Universitario Internacional - Universidad Pablo de Olavide \\ Irene Liberia Vayá | iliberia@us.es \\ Universidad de Sevilla \\ Sara Mansanet Royo|Sara.mansanet@fundacions.uv.es \\ Universitat de València
}

Keywords

"film festival"; "cinema"; "COVID"; "pandemic";

"Valencian Community".

\section{Summary}

1. Introduction

2. Methodology

3. Quantitative data report: FestiMapp 2020 survey

4. Qualitative data report: discussion groups

5. Conclusion

6. References

\section{Abstract}

This paper analyses the impact of the COVID-19 pandemic on film festivals in the Valencian Community against a backdrop of dramatic transformations of the global festival circuit. Firstly, a quantitative approach based on the electronic survey technique was used to collect inputs from all the festivals in the Valencian territory. Secondly, through the qualitative research method of the discussion group, the data and conclusions drawn from the first part of the study were further explored. The main results indicate that, with some variations with regard to their usual dates, a reduction in capacity and film programming, and the adoption of the necessary hygienic-sanitary measures, the vast majority of festivals were able to host their 2020 editions; additionally, and despite the remarkable differences between large, mainstream festivals and those with a smaller size or scope, all of them aim to return to their physical (non-virtual) formats, although applying the lessons of the last year. The pandemic has also highlighted the urgent need for collaboration between festivals, accelerating the creation of the Association of Film Festivals of the Valencian Community.

\section{How to cite this text:}

Samuel Fernández-Pichel, Irene Liberia Vayá, Sara Mansanet Royo (2021): Los efectos de la pandemia de COVID-19 en el ecosistema de festivales cinematográficos de la Comunitat Valenciana, en Miguel Hernández Communication Journal, Vol. 12 (2) pp. 539 a 563 . Universidad Miguel Hernández, UMH (Elche-Alicante). DOI: 10.21134/mhjournal.v12i.1357. 


\section{Introducción}

La irrupción de la pandemia de COVID-19 ha supuesto un momento crítico en el proceso evolutivo de los festivales cinematográficos. Los condicionantes de la llamada 'nueva normalidad' de los tiempos pandémicos desafían la sostenibilidad de la red global de festivales y empujan a los mismos hacia una nueva fase en su devenir histórico (De Valck, 2020; De Valck y Damiens, 2020). Desde la primavera de 2020, este escenario de excepcionalidad ha sido afrontado por los distintos certámenes a lo largo del globo a partir de soluciones locales a problemáticas comunes intentando paliar así los síntomas más evidentes de la actual coyuntura histórica: la crisis de la mercantilización del evento festival, la de sus modos canónicos de exhibición, así como la de su función de acontecimiento comunitario. La interrupción de sus habituales configuraciones espaciales y temporales aboca, en definitiva, a la necesidad de redefinir el concepto y las prácticas asociadas al festival cinematográfico.

Como advierte Vallejo Vallejo (2014, p. 20-23), el “carácter multidimensional” del evento festival ha sido principalmente abordado dentro de la aún reciente pero prolífica sub-disciplina de los estudios de festivales a partir de conceptos propios de las teorías de sistemas y de redes. De este modo, la noción de nodo o zona de tránsito (De Valck, 2007; Iordanova, 2018) se ha empleado para describir los mecanismos de creación de valor y de legitimación cultural en cuyo epicentro se sitúa la acción de los festivales. Según esbozó Nichols (1994) en uno de los textos seminales para este campo de estudio, dicha acción se sustenta sobre un circuito (o red) a través del cual la circulación e intercambio de imágenes queda inserta en las dinámicas económicas, políticas y estéticas de la globalización. Este marco global(izado) reforzaría la "esencia transnacional" de los festivales en oposición a los nuevos localismos o nativismos culturales (Iordanova, 2016).

En el ámbito español, los estudios sobre festivales han adaptado, desarrollado o matizado los marcos teóricos y metodológicos desarrollados en núcleos académicos foráneos ${ }^{1}$. Entre estas contribuciones, podrían citarse la cartografía inicial sobre el panorama de festivales españoles (Jurado Martín, 2004), el estudio sobre la función subalterna de los mismos en el contexto de las industrias culturales (Jurado Martín, 2018; Jurado- Martín y Cortés-Selva, 2018) y sobre su relevancia como plataforma de lanzamiento de nuevos realizadores (Jurado y Nieto, 2014). Se han propuesto, igualmente, acercamientos a los festivales en cuanto alternativas a la dimensión comercial del cine e instigadores de un arte global emergente (Sedeño Valdellós, 2013); aquellos que atienden a su reformulación en la era digital de acuerdo a patrones de "eventificación" y de "cine experiencial" (Vivar, 2018, 2016); y otros que han incidido en el poder legitimador de los festivales para definir las desiguales relaciones entre centros y periferias culturales y geopolíticas (Campos, 2020, 2018).

${ }^{1}$ Vivar Navas aduce que en la universidad española "en muchos casos las contribuciones son réplicas desde el punto de visa [sic] metodológico y temático de lo que ya se ha hecho en el mundo anglosajón, pero trasladado al panorama de festivales españoles" (2016, p. 24). En una línea similar, Jurado Martín (2018, p. 133) alude a las omisiones y carencias de la investigación de esta sub-disciplina en España en contraste con otras propuestas internacionales. 
La perspectiva teórica adoptada para la presente investigación parte de dos vectores principales: por un lado, y en sintonía con los argumentos presentados por de Valck (2016), abogamos por una definición abierta del fenómeno festival, más preocupada por establecer categorías y marcos analíticos aplicables al estudio comparado entre certámenes diversos (en tamaño, alcance, etc.) que por fijar una taxonomía conclusiva sobre los mismos. De manera complementaria, y dadas las idiosincrasias del objeto de estudio (el ecosistema de festivales valencianos), resulta prioritario situar el análisis e interpretación de dicho objeto sobre el continuo entre los "festival-driven events" y los "film-driven events" (De Valck, 2020) o, en una terminología afín, entre los "eventos festivos de interés cultural" y el "evento cultural de intercambio y difusión del audiovisual" (Jurado y Nieto, 2014: 116).

Para algunos/as críticos/as y analistas, la aún breve experiencia de la transformación de los festivales en la era de la COVID-19 acarrea aprendizajes y advertencias que basculan entre el optimismo y las visiones más funestas. En el segundo grupo se sitúan quienes interpretan los ajustes y experimentos en curso como una preocupante sustracción de los múltiples valores y dimensiones del evento festival (Heredero, 2020); o aquellos que ponen el foco en los riesgos derivados de la 'plataformización' desregulada del ecosistema de festivales (Lombardo, 2020). Ello podría desembocar en la celebración de "[a]n event without an event" ("un evento sin evento") (Hobbins-White y Limov, 2020). Esta mutación radical del acto de asistir a un certamen cinematográfico es concebida como un vaciamiento de su carácter ritual, sustituido ahora por una actividad frenética y confusa en las interfaces digitales.

Sin embargo, contra este lamento ante la rápida desmaterialización / desterritorialización de los festivales, se alzan otras voces que inciden en los espacios de oportunidad que surgen de esta encrucijada conceptual y práctica. Para B. Ruby Rich (2020), la nueva fase hacia la que camina el evento festival podría estar caracterizada por un mayor énfasis en la programación y el comisariado en respuesta directa al reto de la virtualización y la lógica algorítmica de las recomendaciones. Girando el foco hacia las audiencias, Brunow (2020) y Petty (2020) desafían la oposición entre lo analógico y lo digital para subrayar el nacimiento de nuevas comunidades de espectadores/as con seno en el ámbito virtual. Se trataría de unos sentidos de comunidad "multimodales" y "performativos" (Brunow, 2020) que, mediante la escenificación de heterogéneas formas de participación, podrían dar origen a un debate renovado acerca de la accesibilidad y los principios de inclusión y atención a la diversidad de los festivales cinematográficos (Whitaker, 2020).

En el Estado español, la adaptación obligada del evento festival reproduce, con algunas inflexiones autóctonas, las dinámicas brevemente esbozadas en los párrafos previos. En el plano organizativo y del asociacionismo sectorial, cabe destacar el nacimiento, en el verano de 2020, de Pantalla, la Federación Estatal de Coordinadoras de Festivales de Cine y Contenidos Audiovisuales. La creación de Pantalla, que a fecha de abril de 2021 aglutina una cifra aproximada de 180 festivales y muestras de cine, ocurre en paralelo, o como estadio previo al surgimiento de iniciativas similares en distintas comunidades autóno- 
mas españolas ${ }^{2}$. Con Pantalla como posible epicentro, las coordinadoras están llamadas a ser agentes o actores principales en esta reconfiguración forzada de la red de festivales, actualizando y expandiendo un espacio de debate y convergencia que ya se había iniciado antes de la pandemia.

Con estos debates teóricos y medidas prácticas de fondo, el presente artículo expone los resultados de una investigación acerca de la incidencia de la crisis de la COVID-19 en un entorno cultural e industrial específico: el ecosistema de festivales cinematográficos de la Comunitat Valenciana. Se recogen en este texto las conclusiones preliminares ya esbozadas en un apartado del Informe FestiMapp 2020 (Fernández, Liberia y Mansanet, 2021), una iniciativa bianual propulsada y financiada por el Institut Valencià de Cultura (IVC) de la Generalitat Valenciana desde el año 2014. El objetivo rector de la investigación se centra en articular, por medio de parámetros concretos, el impacto de la pandemia en los certámenes valencianos a lo largo de 2020, así como en sondear las previsiones de los/las responsables de los festivales acerca de las potenciales transformaciones del ecosistema objeto de estudio. De la observación de dicho objeto de estudio local se han de derivar, en consecuencia, algunas proposiciones encaminadas a contribuir a la redefinición del fenómeno festival cinematográfico en la era pos-COVID.

\section{Metodología}

Los informes bianuales FestiMapp se basan en una encuesta dirigida a los festivales de cine valencianos con un doble objetivo: mantener actualizado el censo de los certámenes en activo en la Comunitat Valenciana y rastrear sus condiciones y necesidades para poder desarrollar políticas públicas adecuadas en el sector. En su última edición se han producido algunas novedades importantes, entre las que destacan la ampliación del cuestionario para dar cabida a las consecuencias de la COVID-19, y el desarrollo, por primera vez, de una investigación cualitativa a través de grupos de discusión.

Respecto a la encuesta, FestiMapp 2020 reúne, reorganizadas, muchas de las variables de las entregas precedentes (logística y estructura, contenidos y temáticas, medición y perfil de las audiencias, etc.), al tiempo que incluye un apartado específico para medir los efectos más relevantes de la pandemia en los festivales del territorio valenciano, y que constituye el objeto de estudio principal de este artículo. Específicamente, se abordan las repercusiones de la COVID en la edición de 2020 y las previsiones para el 2021 en términos de fechas de celebración, presupuesto, número de secciones, contenidos online o actividades paralelas. Asimismo, se pregunta acerca de la posibilidad de que los cambios permanezcan en el tiempo.

El universo de análisis está conformado por la totalidad de los festivales de la Comunitat

${ }^{2}$ Así, a mediados de julio de 2020 se presenta Fescinex - Coordinadora de Festivales de Cine de Extremadura; a finales de agosto lo hace Profestivales 21, unión de los festivales de cabecera andaluces (Huelva, Málaga y Sevilla) y, ya a principios del otoño del mismo año, se da a conocer la coordinadora Illes Balears Film Festivals (IBFF). 
Valenciana. Para ello, en primer lugar se ha creado una base de datos tomando el censo de los anteriores informes FestiMapp, que se ha completado mediante un rastreo manual de festivales. A todos los que integran dicha base de datos se les ha hecho llegar el cuestionario para su cumplimentación telemática, y se ha realizado un seguimiento posterior. De los 77 certámenes contabilizados, han rellenado la encuesta 59, lográndose una tasa de respuesta que asciende al $77 \%{ }^{3}$. A partir de estos datos se ha llevado a cabo un trabajo estadístico descriptivo que pretende servir de soporte para el estudio longitudinal de la situación del sector en los próximos años.

A todo ello se suma una segunda fase cualitativa que se ha basado en el grupo de discusión como técnica de investigación (Infesta Domínguez, Vicente y Cohen, 2012; Parada Dueñas, 2012; Morgan, 1998; Ibáñez, 1991). Atendiendo a los resultados de la encuesta y a las conclusiones alcanzadas, se ha seleccionado un conjunto de festivales que han sido invitados a participar en dos grupos distintos en función de su envergadura, contenidos y características, y siguiendo también la reflexión teórica de los últimos años sobre el ámbito de los festivales de cine. La selección de los certámenes que conforman cada grupo se ha realizado entre los 59 que han respondido a la encuesta FestiMapp 2020, y han quedado conformados del siguiente modo ${ }^{4}$ :

Grupo A: Mostra de València-Cinema del Mediterrani, Festival Internacional de Cine de València Cinema Jove, Festival Internacional de cine de Alicante, DocsValencia, Festival de Cine de l'Alfàs del Pi.

Grupo B: Cortocomenius; Mostra La Ploma. Festival Internacional de Cinema i Cultura per la Diversitat Sexual, de Gènere i Familiar; Quartmetratges; Requena y... ¡Acción!; Semana de Cine de Villena; Skyline Benidorm Film Festival; Cineculpable. Festival Internacional de Curtmetratges de Vila-Real.

La actual situación de crisis sanitaria ha obligado a proceder de manera virtual, por lo que se ha considerado oportuno dividir, a su vez, los grupos de discusión en dos sesiones de dos horas de duración cada una, que han ahondando en temas, problemas y líneas de trabajo extraídos de los resultados de la primera parte del Informe FestiMapp 2020. En este artículo se recogen las conclusiones relacionadas más directamente con los cambios y replanteamientos que ha impuesto la pandemia de COVID-19 a corto y medio plazo, pero también con la voluntad de permanencias y continuidades pese a los obstáculos y la incertidumbre.

${ }^{3}$ No obstante, cabe advertir que no todas las preguntas de la encuesta eran de carácter obligatorio, por lo que este porcentaje desciende en algunos casos, a lo que hay que añadir también la imprecisión de determinadas informaciones y ciertas contradicciones detectadas en los datos aportados por varios festivales.

${ }^{4}$ La nómina definitiva de los grupos se ha visto condicionada por la respuesta de los seleccionados en primer término y también por las agendas de las personas representantes de cada festival. 


\section{Resultados cuantitativos: encuesta FestiMapp 2020}

En primer lugar, y a modo de contextualización, en la siguiente tabla se recogen los 59 festivales participantes en FestiMapp 2020, que incluye tanto certámenes con sección competitiva como muestras de carácter muy diverso.

Tabla 1. Festivales valencianos participantes en la encuesta FestiMapp 2020

\begin{tabular}{|c|c|c|c|c|}
\hline Nombre del festival & Municipio & Provincia & $\begin{array}{c}\text { Mes de } \\
\text { celebración }\end{array}$ & $\begin{array}{c}\text { Nuimero de } \\
\text { ediciones } \\
\text { (hasta 2020) }\end{array}$ \\
\hline $\begin{array}{c}\text { ALMA - Festival } \\
\text { Internacional de } \\
\text { Cortometrajes de } \\
\text { Almassora }\end{array}$ & Almassora & Castelló & Noviembre & 2 \\
\hline Amfibi & El Saler y Sueca & València & Julio & 8 \\
\hline Animalcoi & Alcoi & Alacant & Noviembre & 1 \\
\hline $\begin{array}{c}\text { Bienal Internacional } \\
\text { Dona i Cinema / Mujer y } \\
\text { Cine / Woman \& Film }\end{array}$ & València & València & Enero & 5 \\
\hline Catacumba Film Festival & Godella & València & Octubre/noviembre & 17 \\
\hline $\begin{array}{l}\text { Certamen Autonómico } \\
\text { de Cortos Santa Pola }\end{array}$ & Santa Pola & Alacant & Noviembre & 8 \\
\hline $\begin{array}{l}\text { Certamen de Creación } \\
\text { Audiovisual PROYECTA }\end{array}$ & $\begin{array}{l}\text { Alfara del } \\
\text { Patriarca }\end{array}$ & València & Noviembre & 14 \\
\hline $\begin{array}{c}\text { Cineculpable - Festival } \\
\text { Internacional de } \\
\text { Curtmetratges de Vila- } \\
\text { real }\end{array}$ & Vila-real & Castelló & Noviembre & 23 \\
\hline $\begin{array}{c}\text { Cinema Jove - Festival } \\
\text { Internacional de Cine de } \\
\text { València }\end{array}$ & València & València & Junio & 35 \\
\hline $\begin{array}{c}\text { CortiFestival Cine } \\
\text { Escolar }\end{array}$ & Elx y Alacant & Alacant & Mayo & 3 \\
\hline Cortocomenius & València & València & Octubre & 14 \\
\hline $\begin{array}{c}\text { Cortoons Festival Gandia } \\
\text { - Festival Internacional } \\
\text { de Cortometrajes de } \\
\text { Animación }\end{array}$ & Gandia & València & Octubre & 16 \\
\hline $\begin{array}{c}\text { CortoPilar - Festival } \\
\text { Internacional de } \\
\text { Cortometrajes de Pilar de } \\
\text { la Horadada }\end{array}$ & $\begin{array}{l}\text { Pilar de la } \\
\text { Horadada }\end{array}$ & Alacant & Noviembre & 4 \\
\hline Cortor Festival & El Tormo & Castelló & Agosto & 12 \\
\hline DocsValència & València & València & Noviembre & 5 \\
\hline DocuVir. 20 & $*$ Online & *Online & Junio & 1 \\
\hline
\end{tabular}




\begin{tabular}{|c|c|c|c|c|}
\hline Drets Humans i... Acció! & $\begin{array}{c}\text { Castelló de la } \\
\text { Plana }\end{array}$ & Castelló & Noviembre & 6 \\
\hline $\begin{array}{c}\text { Emove Festival - Festival } \\
\text { Escolar y Universitario de } \\
\text { las Artes Audiovisuales }\end{array}$ & València & València & $\begin{array}{c}\text { *De octubre a } \\
\text { diciembre (1 año) }\end{array}$ & 5 \\
\hline $\begin{array}{c}\text { Erotic \& Bizarre Art Film } \\
\text { Festival }\end{array}$ & Alacant & Alacant & Diciembre & 6 \\
\hline Fantasti'CS & $\begin{array}{c}\text { Castelló de la } \\
\text { Plana }\end{array}$ & Castelló & Noviembre & 10 \\
\hline $\begin{array}{c}\text { FES OH CURT! - } \\
\text { Festival de Curtmetratges } \\
\text { de Nules } \\
\end{array}$ & Nules & Castelló & Febrero & 3 \\
\hline $\begin{array}{c}\text { Festival Cinema Ciutadà } \\
\text { Compromés }\end{array}$ & $\begin{array}{l}\text { *Toda la } \\
\text { Comunitat } \\
\text { Valenciana } \\
\end{array}$ & $\begin{array}{l}\text { *Toda la } \\
\text { Comunitat } \\
\text { Valenciana } \\
\end{array}$ & Octubre-noviembre & 6 \\
\hline $\begin{array}{c}\text { Festival de Cine de } \\
\text { L'Alfàs del } \mathrm{Pi}\end{array}$ & L'Alfàs del $\mathrm{Pi}_{1}$ & Alacant & Julio & 32 \\
\hline $\begin{array}{c}\text { Festival de Cine Sant } \\
\text { Joan d'Alacant }\end{array}$ & $\begin{array}{l}\text { Sant Joan } \\
\text { d'Alacant }\end{array}$ & Alacant & Mayo & 20 \\
\hline $\begin{array}{c}\text { Festival de Curtmetratges } \\
\text { K-lidoscopi }\end{array}$ & Cullera & València & Octubre & 9 \\
\hline $\begin{array}{c}\text { Festival Internacional de } \\
\text { Cine de Alicante }\end{array}$ & Alacant & Alacant & Octubre & 17 \\
\hline $\begin{array}{c}\text { Festival Internacional de } \\
\text { Cine Infantil de València } \\
\text { (FICIV) }\end{array}$ & València & València & Octubre & 6 \\
\hline $\begin{array}{c}\text { Festival Internacional de } \\
\text { Cine de Sax }\end{array}$ & Sax & Alacant & Julio & 14 \\
\hline $\begin{array}{c}\text { Festival Internacional de } \\
\text { Cine Independiente de } \\
\text { Elche }\end{array}$ & Elx & Alacant & Julio & 43 \\
\hline $\begin{array}{c}\text { Festival Internacional de } \\
\text { Cine Pequeño }\end{array}$ & Aspe & Alacant & Agosto & 7 \\
\hline $\begin{array}{c}\text { Festival Internacional de } \\
\text { Curtmetratges de Vinaròs } \\
\text { Agusti Comes }\end{array}$ & Vinaròs & Castelló & Julio & 19 \\
\hline Festival Mostra't Gandia & Gandia & València & Febrero & 5 \\
\hline $\begin{array}{l}\text { Humans Fest }- \text { Festival } \\
\text { Internacional de Cinema } i\end{array}$ & València & València & Febrero & 11 \\
\hline
\end{tabular}




\begin{tabular}{|c|c|c|c|c|}
\hline $\begin{array}{c}\text { Drets Humans de } \\
\text { València }\end{array}$ & & & & \\
\hline $\begin{array}{c}\text { LABdeseries - Festival de } \\
\text { Series de València }\end{array}$ & València & València & Abril & 1 \\
\hline $\begin{array}{c}\text { La Cabina - Festival } \\
\text { Internacional de } \\
\text { Mediometrajes de } \\
\text { València }\end{array}$ & València & València & Noviembre & 13 \\
\hline La Ruta Más Corta & València & València & Diciembre & 8 \\
\hline $\begin{array}{l}\text { Maniatic - Festival } \\
\text { Internacional de Cine } \\
\text { Fantástico de Manises }\end{array}$ & Manises & València & Octubre & 4 \\
\hline $\begin{array}{c}\text { MICE - Mostra } \\
\text { Internacional Cinema } \\
\text { Educatiu }\end{array}$ & València & València & Octubre & 8 \\
\hline $\begin{array}{c}\text { MON-DOC - Mostra } \\
\text { Internacional de Cinema } \\
\text { Documental de } \\
\text { Montaverner }\end{array}$ & Montaverner & València & Noviembre & 12 \\
\hline $\begin{array}{c}\text { Mostra Audiovisual Vila } \\
\text { d'Onda En Xicotet }\end{array}$ & Onda & Castelló & Abril & 3 \\
\hline $\begin{array}{c}\text { Mostra Curts de } \\
\text { Moscatell de Teulada }\end{array}$ & Teulada & Alacant & Agosto & 5 \\
\hline $\begin{array}{c}\text { Mostra de Cinema } \\
\text { d'Ontinyent }\end{array}$ & Ontinyent & València & Octubre/noviembre & 19 \\
\hline $\begin{array}{c}\text { Mostra de Cinema Jove } \\
\text { d'Elx }\end{array}$ & Elx & Alacant & Mayo & 22 \\
\hline $\begin{array}{c}\text { Mostra de Cinema Rural } \\
\text { "Al nostre ritme" }\end{array}$ & Villores & Castelló & Julio & 8 \\
\hline $\begin{array}{l}\text { Mostra de València - } \\
\text { Cinema del Mediterrani }\end{array}$ & València & València & Octubre & 35 \\
\hline $\begin{array}{c}\text { Mostra La Ploma - } \\
\text { Festival Internacional de } \\
\text { Cinema i Cultura per la } \\
\text { Diversitat Sexual, de } \\
\text { Gènere i Familiar }\end{array}$ & València & València & Octubre & 6 \\
\hline $\begin{array}{c}\text { Navidades Sangrientas - } \\
\text { Weekend Horror }\end{array}$ & Alacant & Alacant & Diciembre & 18 \\
\hline Prime the Animation! & València & València & Octubre & $\begin{array}{l}4 * 8 \text { desde } \\
\text { sus inicios }\end{array}$ \\
\hline
\end{tabular}




\begin{tabular}{|c|c|c|c|c|}
\hline & & & & $\begin{array}{l}\text { como } \\
\text { muestra) }\end{array}$ \\
\hline Quartmetratges & Quart de Poblet & València & Octubre & 37 \\
\hline $\begin{array}{c}\text { Rafal en Corto - Festival } \\
\text { Nacional de } \\
\text { Cortometrajes y } \\
\text { Audiovisual de Rafal }\end{array}$ & Rafal & Alacant & Noviembre & 9 \\
\hline Requena y... jAcción! & Requena & València & Junio & 6 \\
\hline RIURAU Film Festival & $\begin{array}{l}\text { Xàbia, Dénia, } \\
\text { Jesús Pobre i La } \\
\text { Xara }\end{array}$ & Alacant & Agosto/septiembre & $\begin{array}{c}10(9+1 \text { de } \\
\text { presentación })\end{array}$ \\
\hline ROS Film Festival & Alacant & Alacant & Noviembre & 3 \\
\hline Rural Film Fest & $\begin{array}{c}\text { Mancomunitat } \\
\text { de l'Alcoià i el } \\
\text { Comtat }\end{array}$ & Alacant & Julio/agosto & 8 \\
\hline $\begin{array}{c}\text { Semana de Cine de } \\
\text { Villena }\end{array}$ & Villena & Alacant & Agosto & 39 \\
\hline $\begin{array}{l}\text { Series Nostrum - Festival } \\
\text { Internacional de Series }\end{array}$ & Altea & Alacant & Noviembre & 1 \\
\hline $\begin{array}{l}\text { Skyline Benidorm Film } \\
\text { Festival }\end{array}$ & Benidorm & Alacant & Abril & 4 \\
\hline $\begin{array}{c}\text { SOL Russian Film } \\
\text { Festival }\end{array}$ & Torrevieja & Alacant & Junio/julio & 6 \\
\hline $\begin{array}{c}\text { XS Festival de } \\
\text { Curtmetratges de Puçol }\end{array}$ & Puçol & València & Octubre & 9 \\
\hline
\end{tabular}

[Fuente: elaboración propia]

\subsection{Repercusión general de la pandemia}

La primera pregunta en torno a la pandemia de COVID-19 es una valoración genérica sobre si esta ha afectado a la celebración de los festivales valencianos en 2020, y fue respondida afirmativamente por 46 de los 59 certámenes, es decir, casi el 78\%. A ello hay que sumar el hecho de que 2 festivales (Humans Fest y Mostra't Gandia) pudieron desarrollar sus ediciones normalmente por haber tenido lugar antes de mediados del mes de marzo, y que 1 festival (Dona i Cinema) no se celebró por ser de carácter bienal y no estar previsto inicialmente para el 2020. Por su parte, del total de los certámenes que participaron en la encuesta, sólo 9 (15\%) señalaron haber desarrollado la edición de 2020 sin modificaciones importantes y no preveían tampoco cambios en 2021. En consecuencia, puede afirmarse que la incidencia de la crisis sanitaria es muy elevada en el ecosistema de festivales de cine de la Comunitat Valenciana.

\subsection{Cambio de fechas y cancelaciones}

Si se pone el foco, en primer lugar, en las repercusiones específicas en términos de fechas, la casuística es variada y depende en gran medida de la época del año en la que se ubica cada festival y su coincidencia con las distintas fases de la pandemia. Concretamente, de los 46 afectados, 18 (39\%) no sufrieron cambios respecto al calendario previsto, mientras que $13(28 \%)$ fueron pospuestos y/o tuvieron que reprogramarse en otras fechas de 2020. 
Por otra parte, $12(26 \%)$ de este grupo de afectados quedaron en suspenso hasta la edición de 2021 y 1 de los certámenes (La Ruta Más Corta) afirmaba que no sabía si podría seguir celebrándose en el futuro (finalmente no se realizó en 2020 y ha tenido lugar en marzo de 2021, aunque su fecha habitual es en diciembre). Además, a los 12 festivales suspendidos hay que añadir otros 10 también cancelados que no respondieron a la encuesta FestiMapp 2020, por lo que la cifra total de certámenes que no se celebraron en 2020 asciende a 22 (sobre 77, casi el 29\%).

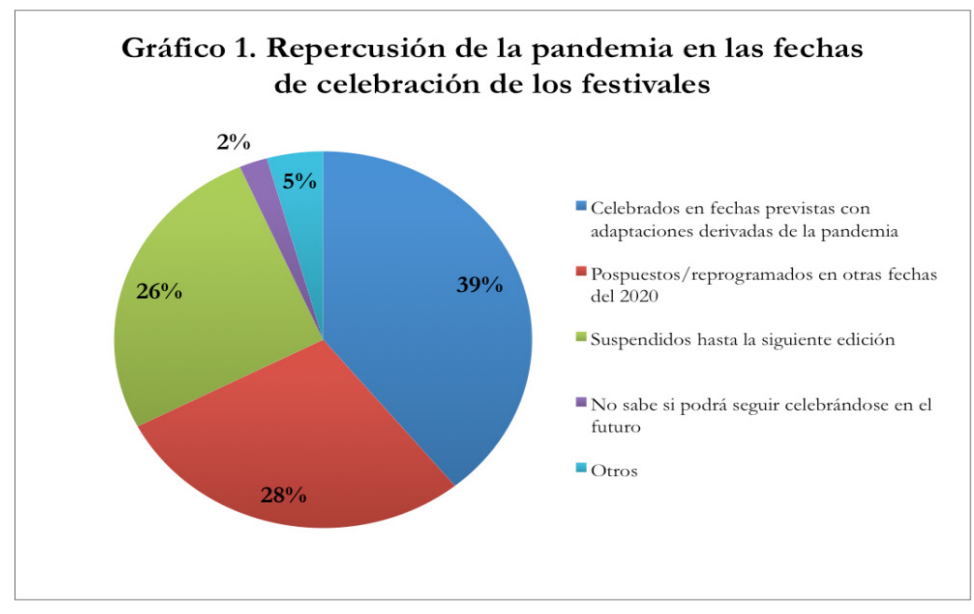

[Fuente: elaboración propia]

\subsection{Variación de los presupuestos}

En términos de presupuestos, llama la atención que 30 festivales del grupo de los 46 afectados aseguran que sus presupuestos no variaron en 2020, lo hicieron muy levemente o incluso experimentaron un aumento. Más específicamente, 19 certámenes (41\%) se sitúan entre los que contaron con un montante igual o superior, mientras que $11(24 \%)$ apenas vieron reducidos sus presupuestos. En el lado contrario se hallan $13(28 \%)$ que sí que señalan reducciones considerables, y $3(7 \%)$ que afirman que estas han sido drásticas.

Preguntados por los motivos de esta merma en el importe con el que cuentan para el desarrollo de sus proyectos, 23 certámenes de los que en mayor o menor medida han experimentado esta rebaja, aducen los siguientes motivos (no excluyentes): menos colaboración privada (17 festivales, esto es, el $74 \%$ de los que se sitúan en este grupo y responden a esta cuestión), menos colaboración pública (12 festivales, el 52\%) y el descenso de la recaudación por taquilla (4 festivales, el 17\%). Dado que muchos de los encuestados ofrecen proyecciones gratuitas o a un coste muy bajo, el ingreso proveniente de las entradas no suele ser una fuente de financiación relevante, y por tanto, son pocos también los certámenes que alegan esta como motivo de reducción presupuestaria. Por lo demás, sí que se observa una mayor merma en los patrocinios privados en comparación con las subvenciones públicas, algo que coincide con los resultados cualitativos extraídos de los grupos de discusión. No 
obstante, cabe puntualizar que los datos referentes a esta variable no son completos ya que de los 27 festivales cuyos presupuestos se vieron afectados en 2020, solo 23 especifican los motivos.

\subsection{Número de películas, secciones y actividades paralelas}

Otro de los ítems a tener en cuenta a la hora de valorar el impacto de la pandemia en los festivales valencianos es el número de películas estrenadas y/o proyectadas durante la edición de 2020. De los 46 certámenes que afirman haberse visto afectados por la crisis sanitaria, cerca de la mitad, 21 (46\%), no reportan cambios o señalan que incluso la cantidad total de filmes fue superior. Por su parte, $11(24 \%)$ indican que apenas se redujo, mientras que la misma cantidad de festivales (otro $24 \%$ ) consignan una bajada considerable del número de películas ofrecidas, y $3(6 \%)$ informan que la disminución fue drástica.

En lo que respecta a la cantidad de secciones, 25 (54\%) de los 46 certámenes mantuvieron el mismo número que en ediciones anteriores y $5(11 \%)$ señalan que en 2020 añadieron alguna nueva. Por el contrario, 6 festivales (13\%) apuntan una reducción, $1(2 \%)$ afirma que, pese a haber eliminado alguna sección, compensó la pérdida con la creación de otras nuevas; y 3 de los encuestados (7\%) explican que llevaron a cabo una reorganización completa de las secciones ofrecidas, principalmente motivada por el paso a la modalidad online ${ }^{5}$.

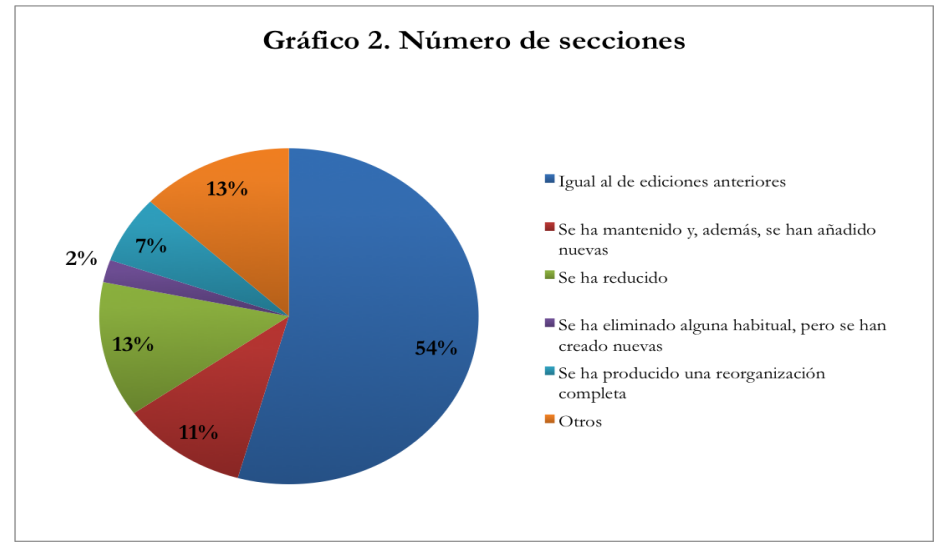

[Fuente: elaboración propia]

Por otro lado, y como también se ha podido corroborar posteriormente en los grupos de discusión, las actividades culturales, formativas y, en general, todas las iniciativas que se celebran paralelamente a las proyecciones, han sido las más perjudicadas por la crisis sanitaria. En concreto, $23(50 \%)$ de los 46 festivales afectados vieron reducidas de manera considerable estas actividades, 19 (41\%) lograron adaptarlas a la nueva situación, mientras que, por su parte, $4(9 \%)$ de estos certámenes optaron por darles más protagonismo para

${ }^{5}$ Los restantes comentan que carecen de secciones o no dan una respuesta clara al respecto. 
compensar la disminución de proyecciones.

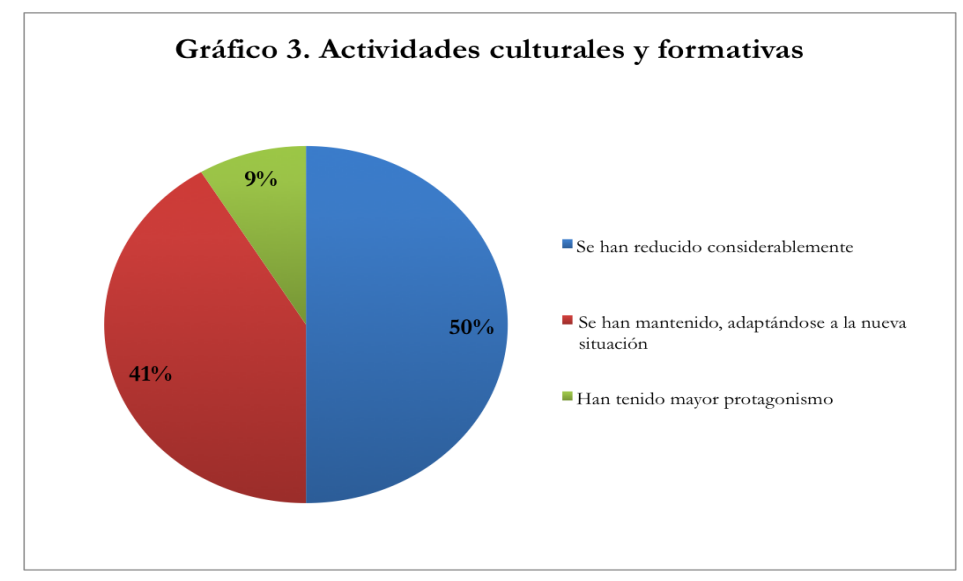

[Fuente: elaboración propia]

\subsection{Actividad online y expectativas a largo plazo}

Otra de las variables importantes para medir las consecuencias de la pandemia en los festivales es el volumen de actividad online en comparación con ediciones anteriores. Los resultados son llamativos porque el $52 \%$ de los certámenes, 24 de 46, aseguran que los contenidos e iniciativas online se mantuvieron durante el 2020 prácticamente igual que en entregas previas (o no preveían modificaciones reseñables en el caso de los que, en el momento de rellenar la encuesta, todavía no habían celebrado su edición de 2020). De la otra mitad de festivales que se han visto afectados por la COVID-19, 7 (15\%) afirman que la actividad online experimentó un leve aumento, 4 (9\%) que este aumento fue considerable y $11(24 \%)$ manifiestan un crecimiento muy considerable de esta parte online.

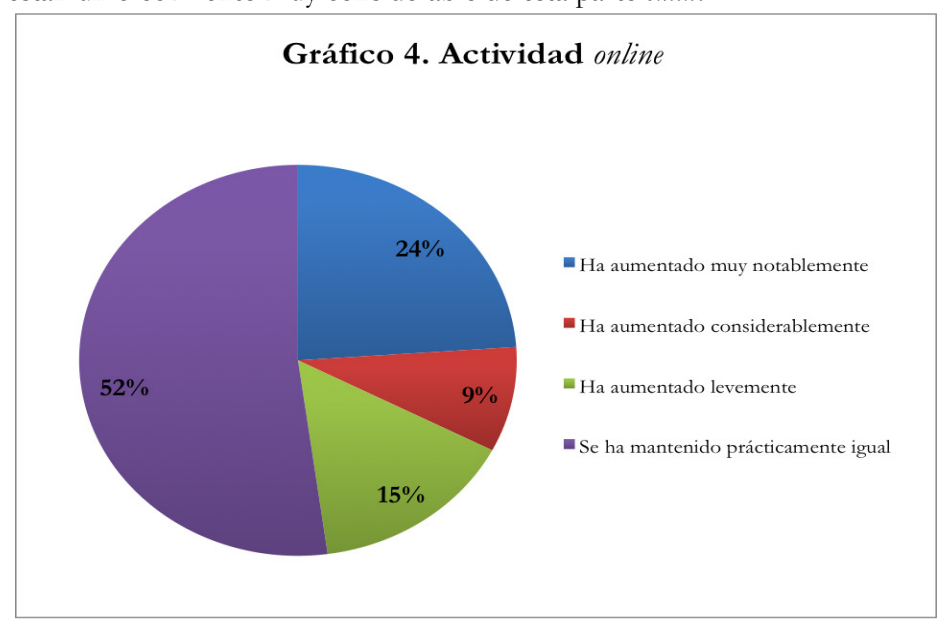

[Fuente: elaboración propia] 
Aunque no se presentan de manera sistematizada, la encuesta también ha permitido recoger, a través de un espacio específico para comentarios cualitativos, otro tipo de consecuencias que la crisis sanitaria ha provocado en los festivales de cine. Entre las más repetidas, se encuentran las siguientes: el rotundo descenso de la presencia internacional de invitados/as y agentes de la industria, la suspensión de entregas de premios para evitar desplazamientos, la disminución de acreditaciones de prensa, la cancelación de actividades para escolares, las importantes reducciones de aforo o la necesidad de algunos festivales de organizar proyecciones al aire libre.

Por último, preguntados sobre la permanencia a largo plazo de los cambios reseñados, este segmento de 46 integrantes del ecosistema valenciano de festivales se muestra confiado mayoritariamente en la vuelta a cierta normalidad. En concreto, 26 certámenes (57\%) niegan el impacto de los cambios de forma permanente y manifiestan su interés por regresar a la situación previa a la pandemia. Por su parte, 13 de ellos (28\%) afirman que las transformaciones no serán esenciales y que el objetivo es poder mantener un formato similar al habitual, al que se añadirían algunas actividades online; 6 certámenes (13\%), por el contrario, auguran un impacto sostenido en el tiempo respecto a las modificaciones apuntadas y advierten del paso a un modelo híbrido que combine la presencialidad con las iniciativas digitales ${ }^{6}$.

\subsection{Primeros festivales del calendario: consecuencias de la pandemia en 2021}

Para finalizar, y como se ha adelantado al comienzo de este epígrafe, 2 festivales de los 59 que respondieron a la encuesta pudieron desarrollar sus ediciones de 2020 con normalidad por celebrarse a principios de año y un tercero no tuvo lugar por ser bienal. Se trata concretamente de Humans Fest, Mostra't Gandia y Bienal Internacional Dona i Cinema. Todos ellos apuntaban en sus respuestas que preveían modificaciones en 2021, aunque también coincidían en su deseo de mantener las fechas habituales, cumpliendo con las adaptaciones necesarias derivadas de la crisis sanitaria. Respecto al resto de indicadores, informaban de un presupuesto igual o superior al acostumbrado y de un número de proyecciones, y también de secciones, equivalente o mayor al de ediciones previas; pero no mostraban la misma opinión respecto a la actividad online ni a la profundidad y permanencia de los cambios inducidos por la COVID-19.

En el momento de cierre del presente artículo ${ }^{7}$, puede confirmarse que los 3 festivales ya han celebrado sus entregas de 2021: Dona i Cinema tuvo lugar entre el 15 y el 23 en enero, con una combinación de proyecciones y actividades presenciales y online; mientras que Humans Fest lo hizo del 8 al 22 de febrero, con una duración de 15 días por primera vez en su

\footnotetext{
${ }^{6} \mathrm{~A}$ estos hay que sumar un festival que ya se celebraba online previamente al estallido de la pandemia, y que no experimentó grandes cambios en este sentido en su edición de 2020.

${ }^{7}$ Este trabajo ha sido finalizado el 25 de abril de 2021. Los datos referentes a la celebración/cancelación/retraso de festivales valencianos con posterioridad a dicha fecha no han sido incorporados.
} 
historia, y un número de proyecciones y de actividades, tanto virtuales como presenciales, también mayor al habitual (que se suman a otras iniciativas desarrolladas desde marzo de 2020). Por su parte, Mostra’t Gandia -un proyecto sociocultural que más allá de cortometrajes, tiene secciones de ilustración, cómic, fotografía y relato-, se vio obligado a suspender la proyección de los cortometrajes semifinalistas y la gala de entrega de premios, previstas ambas actividades a comienzos del mes de febrero, y finalmente han tenido lugar de manera presencial entre el 22 y el 24 de abril.

Como se ha comentado al inicio de este apartado, La Ruta Más Corta no celebró su edición de 2020 en las fechas previstas a causa de las restricciones sanitarias. Se trata de un circuito de cortometrajes proyectados en distintos locales de los barrios más emblemáticos de València, que tiene lugar desde 2013 en el mes diciembre, coincidiendo con el Día Internacional del Cortometraje (21 de diciembre, el día más corto del año). Finalmente, sus responsables optaron por desplazar la edición de 2020 a marzo de 2021. El certamen se ha desarrollado por primera vez durante dos días -en lugar de uno, como es habitual-para así espaciar el público por las restricciones de aforo. Se ha escogido para ello el 26 y 27 de marzo, en un formato híbrido (presencial y online) con un programa de cortometrajes proyectado en tres librerías de tres barrios diferentes de la ciudad y a través de la propia página web del festival.

\section{Resultados cualitativos: grupos de discusión ${ }^{8}$}

Tras los meses de confinamiento y las restricciones sanitarias derivadas de la pandemia por COVID-19, conseguir que el público vuelva a las salas es uno de los mayores retos a los que se enfrentan los festivales de cine, sobre todo los de mayor envergadura.

Preguntados en términos genéricos sobre cómo ha afectado la pandemia a sus festivales, los participantes en los grupos de discusión se muestran unánimes: incertidumbre es la palabra clave. En concreto, de los integrantes del Grupo B, sólo un festival tuvo que suspender la edición de 2020 y otro la celebró de manera online prácticamente al 100\%, mientras que el resto se vio obligado a tomar distintas medidas según los casos: reducción de la programación estrictamente a las proyecciones, cambio de espacios en algunas de las actividades, reprogramación de fechas... y, obviamente, todos adaptaron los aforos e implementaron las medidas higiénico-sanitarias oportunas. Otra coincidencia general que subrayan es la de

${ }^{8}$ Los representantes de los festivales que han participado en los grupos de discusión son: Eduardo Guillot (Mostra de València-Cinema del Mediterrani), Carmen Losada (Festival Internacional de Cine de València Cinema Jove), Vicente Seva (Festival Internacional de Cine de Alicante), Pau Montagud (DocsValència), Vicent Escrig (Festival de Cine de l'Alfàs del Pi), Pau Romero (Cortocomenius); Luis Noguerol (Mostra La Ploma. Festival Internacional de Cinema i Cultura per la Diversitat Sexual, de Gènere i Familiar), Ramón Alfonso (Quartmetratges); Paula García (Requena y ¡Acción!); Antonio V. Martínez (Semana de Cine de Villena); Beatriz Hernández (Skyline Benidorm Film Festival); Sonia de la Vega (Cineculpable. Festival Internacional de Curtmetratges de Vila-Real). Aprovechamos este espacio para agradecerles de nuevo públicamente su colaboración. 
considerar que una de las principales pérdidas del año ha sido el hecho de no haber podido traer a profesionales invitados/as y llevar a cabo coloquios con el público.

Los integrantes del Grupo A, por su parte, ofrecen experiencias contrapuestas. Todos ellos vieron afectadas sus ediciones de 2020 , pero con resultados muy distintos en algunos casos. Cinema Jove fue trasladado de sus fechas originales (junio) a diciembre y, aunque se pudo lograr la presencialidad, fue necesaria la supresión de las galas: "hubo que improvisar una entrega de premios y no se celebraron todas las secciones habituales", según indica su representante'. Por contra, el Festival de l'Alfàs del Pi, previsto para el mes de julio, se celebró con normalidad en las fechas habituales, "todo presencial, con planes de contingencia exhaustivos" y sin contar con ninguna plataforma digital.

Mostra de València mantuvo, a su vez, sus fechas previstas del mes de octubre, adaptándose a las medidas restrictivas. En palabras de su responsable, "nos agarramos a la frase de José Luis Rebordinos, director del festival de San Sebastián, que dijo: 'San Sebastián será presencial o no será'. Con toda la modestia, pensamos que, si ellos o Venecia podían, porqué nosotros no, asumiendo esas mismas medidas sanitarias y restricciones". Sin embargo, DocsValència sí que tuvo que retrasar sus fechas hasta la segunda mitad de diciembre y desarrolló un festival 100\% online. El certamen se articula en exhibición, formación e industria y, según su director, "la propia naturaleza del festival nos impedía celebrar una edición híbrida". Asimismo, el Festival Internacional de Cine de Alicante movió sus fechas de mayo a octubre, celebrándose en este caso en un formato híbrido. Los encuentros con el público, las charlas y las masterclass se realizaron online con gran éxito de audiencia.

\subsection{Actividad online}

Pese a lo que pudiera esperarse, y como se ha puesto de manifiesto en los resultados obtenidos a través de la encuesta, la actividad online de los festivales valencianos no aumentó exponencialmente en 2020. Este hecho se reafirma especialmente en el caso de los festivales de menor envergadura participantes en los grupos de discusión, que se muestran particularmente rotundos en la importancia de la presencialidad: algunos, en parte, por falta de logística y recursos para reaccionar con velocidad trasladando su programación al formato online, y otros por la incompatibilidad de esta vía con los propios principios que rigen sus festivales. Hay aquí una conexión clara con la misión social que les define, y que se resume en proporcionar un servicio a la ciudadanía, sobre todo a aquella que experimenta mayores dificultades para acercarse a los contenidos audiovisuales que ofrecen. Asimismo, la postura que mantienen respecto a esta cuestión es coherente con la centralidad del factor sociabilidad en este tipo de certámenes, algo que refuerza la concepción de festival de cine como evento festivo y encuentro social.

${ }^{9}$ Los testimonios de los representantes de cada festival han sido modificados ligeramente para adaptarlos al lenguaje escrito, sin alterar en ningún caso su sentido. 
Aunque no resulte tan obvio a priori, también en el caso de los festivales de mayor envergadura hay una coincidencia en defender la presencialidad como su razón de ser fundamental. Por ello, pese a que la mayoría tuvo acercamientos en 2020 a actividades online por la situación pandémica, el camino que trazan en el futuro es de retorno a la máxima presencialidad posible.

No obstante, pese a la férrea voluntad de 'volver a la normalidad', la mayoría valora también las nuevas posibilidades que abre el territorio virtual. Especialmente significativo resulta a este respecto el caso de Mostra La Ploma, el festival del Grupo B que en 2020 derivó las proyecciones a formato online, y que subraya la utilidad y el aprendizaje extraído en estos términos: la modalidad virtual les ha permitido "llegar donde antes no llegaban", pero, más allá, les "ha hecho descubrir que puede haber proximidad en lo online". Algunas actividades como la realización de debates virtuales en colaboración con la Universitat de València, y el interés manifestado por espectadores/as de países latinoamericanos o europeos en su certamen, les ha cambiado mucho la percepción: "es algo muy bonito, son señales que te llevan a replantear ciertas cosas de cara a nuevas ediciones, sin contar el hecho de que la diversificación de públicos ha sido una realidad a nivel geográfico, pese a que nuestra plataforma sólo permitía acceder desde España” (Mostra La Ploma).

Por su parte, todos los festivales del Grupo A reconocen que el formato online ha llegado para quedarse y, lejos de ser una amenaza, las plataformas de streaming se pueden convertir en aliadas a la hora de ofrecer la programación al público y convivir así con los festivales, de manera que estos puedan salir fortalecidos. Pero para ello se necesita partir de una reflexión conjunta y profunda de todos los agentes implicados, y aunar esfuerzos para estar a la altura de todas las transformaciones que se están produciendo.

\subsection{Gestión de audiencias en el entorno online}

En la búsqueda de esta buena convivencia y de lograr los mejores resultados en las actividades presenciales y online, surgen algunas dudas tales como de qué manera contabilizar las audiencias de las actividades y proyecciones que se ofrecen en formato digital y si las cifras resultantes son del todo fiables. Este es un aspecto que preocupa a los festivales de gran formato especialmente, al igual que delimitar qué se considera público de un festival. Es decir, ¿cualquier espectador o espectadora que acuda a cualquier actividad desarrollada por el festival, aunque no sea cinematográfica, es susceptible de ser considerado/a audiencia de dicho festival? Por otro lado, también se plantea la cuestión de cómo se contabilizan los visionados online en las plataformas de streaming. Según indica el director artístico de Mostra de València, "hay plataformas que con 1 minuto dentro de la proyección lo considera como visionado". Esto hace muy complicado valorar si ciertamente la programación está teniendo una buena audiencia real y una buena acogida por parte del público, más allá de contabilizar la cantidad de clics dados a cada película.

Así, aunque parece claro que la cultura sin público carece de sentido, centrar los esfuerzos en una competición feroz por las audiencias no puede constituir la razón de ser última de 
los festivales de cine. A este respecto, algunos de los integrantes del Grupo A, como Mostra de València o DocsValència, señalan que han desarrollado en sus últimas ediciones diferentes estudios y análisis de públicos. De hecho, este último festival realizó una encuesta exhaustiva sobre los hábitos de sus espectadores/as, llegando a generar en 2020 un proveedor propio de streaming para poder tener una medición ad hoc de la respuesta de su audiencia en un entorno online. Según su director, resulta fundamental conocer hasta qué punto el público es recurrente y fiel al festival. Por su parte, Mostra de València encargó ya en su edición de 2019 un estudio de espectadores/as, cineastas y prensa acreditada en el que se valoraban diferentes aspectos organizativos y de programación. En conclusión, según las inquietudes y experiencias de los certámenes participantes en los grupos de discusión, parece evidente que los festivales necesitan un estudio cuantitativo, pero sobre todo cualitativo de públicos, si quieren adecuarse a sus demandas y necesidades, además de atraer a nuevas audiencias.

\subsection{Impulso asociativo}

En anteriores informes de FestiMapp, se venía apuntando reiteradamente la necesidad de crear una coordinadora o asociación que aglutinase el mayor número posible de certámenes cinematográficos de la Comunitat con el fin, por un lado, de constituir una voz definida ante la Administración Pública y, por otro, de disponer de una plataforma de comunicación, coordinación y ayuda mutua. La encuesta de FestiMapp 2020 no hace sino reforzar esta postura, ya que 56 de los 59 festivales que han participado en ella responden afirmativamente a la pregunta sobre la conveniencia de poner en marcha una entidad de este tipo.

El impulso final dado para la creación de FESCICOM (Asociación de Festivales de Cine de la Comunitat Valenciana) es consecuencia de todo lo acontecido en 2020. Los problemas nuevos relacionados con la pandemia, unidos al hecho de que por primera vez (y por las mismas circunstancias) se ha creado una Federación Estatal de Coordinadoras de Festivales de Cine (Pantalla), ha empujado a poner en marcha una asociación propia y a cohesionar, de esta manera, a todos los certámenes que tienen lugar en el territorio valenciano.

No obstante, desde el primer momento, en la celebración de los grupos de discusión se ha observado una dicotomía clara respecto a esta cuestión: el Grupo B, conformado en su mayoría por festivales de menor envergadura, perciben la asociación como algo ajeno, como algo "de los grandes", aunque la consideren una iniciativa muy positiva que les puede aportar beneficios. Por su parte, los integrantes del Grupo A, en el que se encuentran varios miembros de la Junta Directiva provisional de la asociación, apelan a la necesidad de que todos los certámenes, tengan el tamaño que tengan, se impliquen. Según el director artístico de Mostra de València, "lo mejor que puede hacer un festival pequeño para saber cómo

${ }^{7}$ Maldita Castilla es un claro referente para Blasphemous, pero no es el único, existen otros muchos títulos que han sabido adaptar en forma videolúdica el pasado y determinadas estéticas asociadas a la forma de videojuego, como por ejemplo El Cid (Dro Soft, 1987), Don Quijote (Dinamic, 1987), El Capitán Trueno (Dinamic, 1989), o Curro Jiménez (Zigurat, 1989). Sobre este aspecto véase Acosta Riego, D., y Navarrete Cardero, L. (2017). Spain ludica. La imagen romántica de España en el videojuego. Barcelona: Editorial UOC. 
funcionará la asociación, es precisamente asociarse. De hecho, teniendo en cuenta que en la Comunitat hay bastantes más festivales de pequeño formato, cuantos más se unan a la asociación, esta va a ser más fuerte. [...] Es en las problemáticas comunes donde tenemos que encontrarnos todos".

En la misma línea, la directora de Skyline Benidorm Film Festival, integrante del Grupo de Discusión B y también de la Junta Directiva provisional de la asociación, apunta como objetivo principal de esta constituir una herramienta para ayudarse entre todos y sumar, además de unirse a Pantalla. En el momento de celebración de los grupos de discusión, FESCICOM sumaba ya 35 confirmaciones, una cifra -susceptible de aumentar- que la convertirá, presumiblemente, en una de las coordinadoras regionales más potentes cuando se integre en Pantalla.

\subsection{Expectativas a medio y largo plazo}

Los festivales que componen el Grupo A se enfrentan a problemáticas distintas de cara a sus próximas ediciones. En concreto, parece que la programación de 2021 se presenta algo complicada en estos festivales grandes que ofrecen en su selección estrenos autonómicos o nacionales, ya que, según explica el responsable de DocsValència, "nos viene un cuello de botella de programación brutal. Se van a juntar los estrenos de 2021 más todos aquellos que decidieron aplazarse. Hay festivales que tienen que adaptarse para sobrevivir y hacer un ejercicio de introspección para saber qué es necesario mantener y de qué podemos prescindir".

Ante esta difícil situación, reconocen en algunos casos haber adaptado sus bases de 2021 para hacer más accesible la selección. El Festival Internacional de Cine de Alicante, por ejemplo, ha cambiado los requisitos "como algo excepcional: las películas españolas de sección oficial pueden haberse estrenado en salas comerciales con anterioridad. Si vetamos las estrenadas en salas, nos llegaría muy poco material este año". En cualquier caso, todos reconocen abiertamente que la experiencia de 2020 ha sido positiva a pesar de las circunstancias.

Todo lo señalado hasta el momento reafirma a los festivales en su intención de mantener la máxima presencialidad en 2021, pero incorporando los aprendizajes del 2020. Como señala el coordinador de Mostra La Ploma, "la fórmula tiene que ser volver a lo físico: lo esencial será el encuentro con el público y los profesionales en persona, compartir en directo el proceso de creación. Aunque hay pequeñas cosas que nos ha enseñado este año y que podemos ir incorporando. En definitiva, abrir nuevas vías complementarias, no sustitutivas". Precisamente en esas enseñanzas extraídas de la pandemia insiste el responsable de Quartmetratges cuando habla de la vía online como oportunidad, como "forma de abrir miras", y también en este punto parte de los integrantes del Grupo B apelan a la necesidad de formación de los equipos que desarrollan los festivales y al papel clave que aquí puede y debe jugar la Administración Pública.

Como reiteran los festivales de menor tamaño, los proyectos salen adelante gracias al esfuerzo y enorme trabajo de personas que en su mayoría no cobran por esas funciones y 
que, muy a menudo, no tienen ninguna formación en gestión cultural. En esencia, reclaman acciones formativas en administración y gestión, comunicación y promoción, captación de públicos, dirección de equipos, también algún tipo de formación técnica, y -muy importante- talleres y campañas de sensibilización sobre derechos de autor y acerca de la necesidad de cumplir la legalidad establecida para la proyección pública de contenidos audiovisuales. Además de mejorar el resultado de los festivales, como señalan algunos, la formación es una manera de motivar y de que, quienes ahora trabajan voluntariamente, vayan asumiendo responsabilidades, con las consecuencias positivas que ello tendría para el fortalecimiento de los propios festivales.

\section{Conclusiones}

El marco de incertidumbre que han referido los/las responsables de los festivales participantes en este estudio determina, igualmente, cualquier intento por delimitar el curso inmediato de este fenómeno cultural, tanto en sus declinaciones específicas en el caso de estudio abordado en estas páginas como en escalas geográficas más amplias. No obstante, se pueden conjeturar y extrapolar algunas líneas de desarrollo a partir de las informaciones e indicios aquí recabados que, a manera de desafíos futuros, atañen a la sostenibilidad de los festivales cinematográficos.

En primer lugar, la singularidad del momento histórico invita a potenciar la reflexión colectiva ya iniciada. La disrupción de la antigua normalidad ha propiciado una conciencia más urgente acerca de la necesidad del asociacionismo en el sector (de ahí el nacimiento de nuevas coordinadoras regionales, como FESCICOM en el caso valenciano, y una estatal en España) que debería ir también acompañado de un debate -a la vez teórico y deontológicoacerca de las múltiples manifestaciones del objeto festival cinematográfico. El criterio de la diversidad debería actuar en esta fase crítica y reflexiva de acuerdo a un contexto de aplicación extenso: de los agentes involucrados en los debates a la reconsideración y ampliación del concepto de audiencias como sujetos-usuarios/as-beneficiarios/as, pero también como "productores de valor" (Vivar Navas, 2016, p. 92-93).

Como extensión de lo anterior, resulta conveniente redefinir en términos claros tanto el anclaje territorial de los festivales como su función social en este nuevo escenario. Si la pandemia ha supuesto un quiebre -aún desconocemos si temporal o definitivo- a los hábitos de movilidad e intercambios propios de la era global, ¿en qué medida afectará ello a los mecanismos de identificación y a la conformación de comunidades en torno al festival como evento? ¿Para qué públicos y bajo qué requisitos y expectativas de participación? ¿Se reforzará la presencia en entornos digitales y la consecuente desmaterialización o hibridación de los festivales? ¿De qué manera se compensará el déficit detectado en la organización de actividades culturales y formativas en y más allá de las fechas concretas de celebración de los certámenes?

Una última vía de exploración concierne tanto al modelo de gestión como a la investigación académica de los festivales cinematográficos, con sus matizaciones en cada esfera de acción. El paradigma dominante desde al menos la última década y media se ha sustentando de 
manera operativa (gestión) o aplicada (estudios académicos) en la lógica de las redes y los flujos, de los dividendos activos y plusvalías conceptuales derivados del capital cultural, de las visiones socio-económicas con réditos intelectuales, de las estrategias de marca y emprendimiento urbanos que derivan parte de su legitimación de la atención prestada desde ámbitos universitarios. ¿Qué ocurre cuando la sostenibilidad completa del paradigma (de prácticas y posicionamientos teóricos) entra en crisis? ¿Cuándo la pausa es impuesta por un actor inesperado (un organismo apenas detectable) que obliga a la distancia e interrumpe la velocidad del flujo global?

Según indica De Valck (2020), la situación (pos)pandémica requiere del uso de nuevas herramientas analíticas que han de venir necesariamente precedidas de la observación atenta de las mutaciones en el ámbito profesional de los festivales. La combinación de estudios de caso e investigaciones de recogida de datos a gran escala - sugerida por la propia autora como posible enfoque metodológico futuro- quizá deba virar en pos de un énfasis aun mayor en los públicos en cuanto colectivos activos y sustanciales para la reconfiguración de los festivales. Se caminaría, de esta forma, hacia una profundización en los factores de autonomía, participación, diversidad y soberanía cultural más allá de los tradicionales centros irradiadores de la cultura y la lógica (des)reguladora de los mercados en red.

\section{Bibliografía}

Brunow, D. (2020). Come together? Curating communal viewing experiences for hybrid and online film festivals. NECSUS, Autumn 2020 (Festival Reviews - Special Dossier: Covid 19, Eds. M. de Valck y A. Damiens). Recuperado de: https://bit.ly/3eCGFTh

Campos, M. (2018). Lo (trans)nacional como eje del circuito de festivales de cine. Una aproximación histórica al diálogo Europa-América Latina. IMAGOFAGLA: Revista de la Asociación Argentina de Estudios de Cine y Audiovisual 17, Buenos Aires, abril, pp. 11-40. Recuperado de: https://bit.ly/3h7JYSo

Campos, M. (2020). Tensiones en el circuito cinematográfico internacional: modelo para el estudio de los festivales latinoamericanos. Comunicación y medios 29(42), Santiago de Chile, diciembre, pp. 72-84. Recuperado de: https://bit.ly/3dcBR5K

De Valck, M. (2007). Film Festivals. From European Geopolitics to Global Cinephilia. Amsterdam: Amsterdam University Press.

De Valck, M. (2016). Introduction: What is a film festival? How to study festivals and why you should. In M. de Valck, B. Kredell \& S. Loist (Eds.), Film Festivals: History, Theory, Method, Praxis (pp. 1-11). New York: Routledge.

De Valck, M. (2020). Vulnerabilities and Resiliency in the Festival Ecosystem: Notes on Approaching Film Festivals in Pandemic Times. In P. D. Keidl, L. Melamed, V. Hediger and A. Somaini (Eds.), Pandemic Media: Preliminary Notes Toward an Inventory (pp. 125-135). Lüneburg, Germany: Meson Press. 
De Valck, M. y Damiens, A. (2020). Film festivals and the first wave of COVID-19: Challenges, opportunities, and reflections on festivals' relations to crises. NECSUS, Autumn 2020 (Festival Reviews - Special Dossier: Covid 19, Eds. M. de Valck y A. Damiens). Recuperado de: $\underline{\text { https://bit.ly/3uFKGfc }}$

Fernández Pichel, S., Liberia Vayá, I. y Mansanet Royo, S. (2021). FestiMapp 2020. Censo de festivales de cine en la Comunidad Valenciana (edición bilingüe). València: Institut Valencià de Cultura.

Heredero, C. F. (2020). Festivales en tiempos de pandemia (editorial). Caimán Cuadernos de Cine, 30 de septiembre. Recuperado de: https://bit.ly/2RaHt8S

Hobbins-White, P. y Limov, B. (2020). SXSW, Amazon, and the difficulty of staging an exclusive event online. NECSUS, Autumn 2020 (Festival Reviews - Special Dossier: Covid 19, Eds. M. de Valck y A. Damiens). Recuperado de: https://bit.ly/3o6Dx53

Ibáñez, J. (1991). El grupo de discusión: fundamento metodológico y legitimación epistemológica. En M. Latiesa (Ed.), El pluralismo metodológico en la investigación social (pp. 53-82). Granada: Universidad de Granada: Ensayos típicos.

Infesta Domínguez, G.; Vicente, A. y Cohen, L. (2012). Reflexiones en torno al trabajo con grupos de discusión en ciencias sociales. Intersticios: Revista sociológica de pensamiento crítico 6(1), Madrid, diciembre, pp. 233-244. Recuperado de: https://www.intersticios.es/article/ view/8899

Iordanova, D. (2016). Foreword: The Film Festival and Film Culture's Transnational Essence. In M. de Valck, B. Kredell \& S. Loist (Eds.), Film Festivals: History, Theory, Method, Practice (pp. xi-xvii). London: Routledge.

Iordanova, (2018). The Film Festival: Principal Node in Film Culture. Frames Cinema Journal 13, mayo. Recuperado de: https://bit.ly/3fe4dwu

Jurado-Martin, M. (2004). La Mostra de Valencia en el panorama nacional de festivales de cine. Valencia: Generalitat Valenciana, Fundación Municipal de Cine de la Mostra de Valencia y Ayuntamiento de Valencia.

Jurado-Martin, M. (2018). Propuesta de categorización de festivales de cine (Estudio de caso en España). Miguel Hernández Communication Journal 9, Elche, pp. 131-160. Recuperado de: https://bit.ly/3h5q0aV

Jurado-Martín, M. y Cortés-Selva, L. (2018). Madridimagen: tras las huellas del último festival de cine de fotografía. Discursos fotográficos 14(25), Londrina, julio/agosto, pp. 220-249.

Jurado-Martín, M. y Nieto-Martín, A. (2014). Nuevas propuestas, viejos circuitos: El papel 
de los festivales de cine españoles en la consolidación de los nuevos realizadores. Secuencias: revista de historia del cine 39, Madrid, junio, pp. 100-122. Recuperado de: https://bit. ly/3h9HnqU

Lombardo, M. J. (2020). Un festival en tiempos de pandemia. Diario de Sevilla, 12 de noviembre. Recuperado de: $\underline{\text { https: / / bit.ly/3y3AHCs }}$

Morgan, D. L. (1991). The Focus Groups Guide Book. Newbury Park: Sage.

Nichols, B. (1994). Gobal Image Consumption in the Age of Late Capitalism. East-West Film Journal 8(1), Hawai, January, pp. 68-85.

Parada Dueñas, F. J.(2012). Premisas y experiencias: análisis de la ejecución de los grupos de discusión online. Encrucijadas: Revista Crítica de Ciencias Sociales 4, Salamanca, diciembre, pp. 95-104. https://bit.ly/3fcCxbC

Petty, S. (2020). Story within a story: Vues d'Afrique in the time of COVID-19. NECSUS, Autumn 2020 (Festival Reviews - Special Dossier: Covid 19, Eds. M. de Valck y A. Damiens). Recuperado de: https://bit.ly/2R3s $3 \mathrm{mY}$

Rich, B. R. (2020). When Is an Editorial not an Editorial? The COVID-19 (Quarantine, Era 1) Edition. Film Quarterly 73 (4), pp. 5-10.

Sedeño Valdellós, A. (2013). Globalización y transnacionalidad en el cine: coproducciones internacionales y festivales para un cine de arte global emergente. Fonseca Journal of communication, Monográfico 2, Salamanca, junio, pp. 285-303. Recuperado de: https://bit. $\underline{\operatorname{ly} / 3 g \text { TcuZ0 }}$

Vallejo Vallejo, A. (2014). Festivales cinematográficos. En el punto de mira de la historiografía fílmica. Secuencias: revista de historia del cine 39, Madrid, abril, pp. 13-42. Recuperado de: https://bit.ly/3gVs7is

Vivar Navas, R. (2016). Los festivales de cine en la era de los new media. Una perspectiva lúdica sobre las fiestas del cine y sus públicos. (Tesis doctoral). Universidad de Granada, Granada.

Vivar Navas, R. (2018). Las fiestas del cine: nostalgia, exclusividad y ocasión en el cine-evento en España. Secuencias: revista de historia del cine 47, Madrid, abril, pp. 33-54. Recuperado de: http://dx.doi.org/10.15366/secuencias2018.47.003

Whitaker, J. A. (2020). San Luis Obispo International Film Festival: The social responsibility of redesigning an American film festival. NECSUS, Autumn 2020 (Festival Reviews - Special Dossier: Covid 19, Eds. M. de Valck y A. Damiens). Recuperado de: https://bit. ly/3f9cnX1 


\section{Agradecimientos:}

La realización de esta investigación fue posible gracias a la financiación recibida por parte del Institut Valencià de Cultura (IVC) para la elaboración del censo de festivales de la Comunitat Valenciana (FestiMapp 2020). Nos gustaría agradecer en especial a Dora Martí (IVC) su ayuda durante el intenso proceso de mapeo y contacto con los certámenes. Expresamos igualmente nuestra gratitud hacia los/las responsables y/o miembros de la organización de los mismos, quienes atendieron a nuestro requerimiento completando las encuestas y/o respondiendo a nuestras llamadas. Mención particular merecen los/las integrantes de los grupos de discusión (listados en el cuerpo del artículo), que nos dieron su tiempo y a partir de cuyos testimonios pudimos enriquecer los resultados y reflexiones expuestos en estas páginas. 


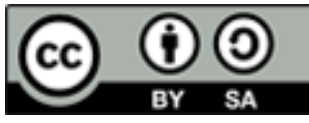

Licencia Creative Commons Miguel Hernández Communication Journal mhjournal.org

\section{Cómo citar este texto:}

Samuel Fernández-Pichel, Irene Liberia Vayá, Sara Mansanet Royo (2021): Los efectos de la pandemia de COVID-19 en el ecosistema de festivales cinematográficos de la Comunitat Valenciana, en Miguel Hernández Communication Journal, Vol. 12 (2) pp. 539 a 563 . Universidad Miguel Hernández, UMH (Elche-Alicante). DOI: 10.21134/mhjournal.v12i.1357 